\title{
The Big Idea: Iron-dependent inflammation in venous disease and proposed parallels in multiple sclerosis
}

\author{
Professor Paolo Zamboni
}

J R Soc Med 2006;99:589-593

This article is based on a lecture presented at the Tripartite Meeting, EVF-AVF-UKVF, Royal Society of Medicine, London, 1 July 2006.

\section{IRON DEPENDENT INFLAMMATION IN CVD}

Impaired venous drainage of the lower extremities, mainly due to venous reflux or to venous outflow obstruction, leads to a cascade of pathological events clinically graded by the clinical class $(\mathrm{C})$ of the CEAP classification (Clinical, aEtiological, Anatomical, Pathophysiological) of chronic venous disease $(\mathrm{CVD}){ }^{1}$ Varicose veins are the most frequent clinical sign in class C2. When oedema complicates varicose veins, the clinical picture is graded as C3. Pigmentation, lipodermatosclerosis and other skin changes are classified as $\mathrm{C} 4$. A small but significant number of the affected patients develop venous ulcers. Healed ulcers are classified as $\mathrm{C} 5$, whereas active ulcers are $\mathrm{C} 6$. Altered venous haemodynamics are a necessary but not exclusive element for explaining progression along the clinical classes to the point of skin lesion. In 1982, Browse and Burnand ${ }^{2}$ observed a peri-capillary fibrin deposition and speculated that cuffs act as a barrier to oxygen diffusion and nutrients, resulting in epidermal cell death. This mechanism of tissue injury has not yet been demonstrated. The fibrin cuff may be more properly considered a scaffold for tissue reparative processes. The cuff contains fibrin, but also laminin, fibronectin, tenascin, and types I and III collagen, encircling the dilated capillary vein ${ }^{3}$ (Figure 1A). The decline of the fibrin cuff theory over the last twenty years has led to investigation of other factors emphasizing inflammatory mechanisms as amplifiers of the insufficient venous drainage. Recent studies demonstrate a pivotal role for tissue iron accumulation in inducing and maintaining inflammation in CVD. ${ }^{4-9}$

Iron deposits in CVD cause readily visible brownish dermal areas which sometimes precede, but always surround, ulcers. The origin of increased leg iron stores is extravasation of red blood cells (erythrocytes) in conditions of significant venous stasis. Erythrocytes are degraded by the interstitial macrophages, with the released

Vascular Diseases Center, University of Ferrara, 44100 Ferrara, Italy

E-mail: zmp@unife.it iron incorporated into ferritin. Over time, with increasing overload of iron, the structure of ferritin changes to haemosiderin. ${ }^{4-9}$ In 1988, Ackermann found a twenty-fold higher average concentration of iron in lower limbs affected by venous ulcers as compared to the upper arm of the same subjects. ${ }^{8}$ The phenomenon of leg haemosiderin deposits seems to be significant for the entire body, since this protein has been demonstrated in the urine of patients affected by CVD. ${ }^{9}$

Increased iron stores and interstitial protein extravasation are potent chemo-attractants and presumably represent the initial underlying chronic inflammatory signal responsible for white blood-cells recruitment and migration in the matrix (Figure 2B). In 1988, Coleridge-Smith observed leukocytes trapped in the venous microcirculation secondary to venous hypertension. This work paved the way to the investigation of the relationship between CVD and inflammation. ${ }^{10}$ The mechanism of white cell migration in the subcutaneous matrix was further elucidated by studies of the expression of adhesion molecules in a model of venous hypertension. Several studies confirmed the expression of these molecules, including ICAM, VCAM and selectins. ${ }^{11,12}$ Such adhesion molecules block circulating white cells on the vein wall and facilitate transmigration into the tissue. The predominant cells migrating into the extra-cellular matrix are macrophages and T-lymphocytes. ${ }^{12}$

Macrophages take up iron accumulated in the tissue and store it in intracellular ferritin-like structures (Figure 3B). Intra- and extra-cellular overload of iron in the tissue could potentially be dangerous for generation of free radicals due

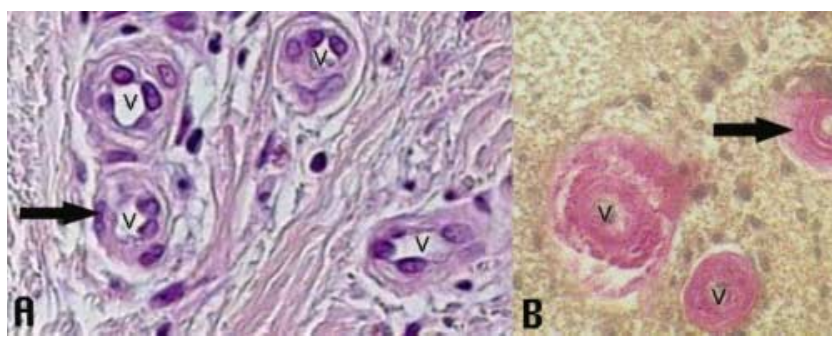

Figure 1 Panel A, classic fibrin cuffs (arrow) thicken veins (v) in a venous ulcer bed, $40 \mathrm{x}$. Panel B, fibrin cuffs (arrow) encircles proliferated thick walled veins (v) in a peri-ventricular MS plaque, $30 \mathbf{x}$. Panel A is courtesy of Professor Caggiati, Rome, Italy. Panel B is modified from Adams CW. A Colour Atlas of Multiple Sclerosis. London: Wolfe Med, 1989. 


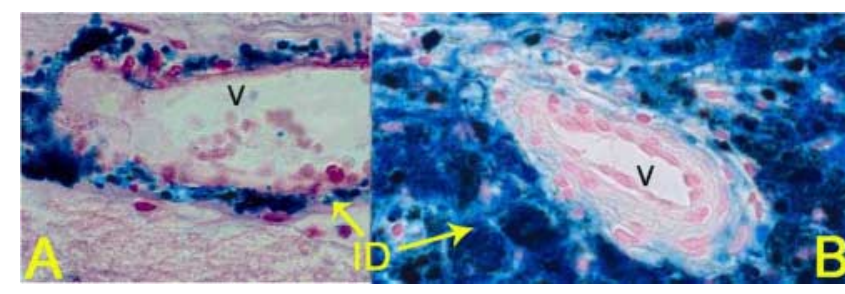

Figure 2 Panel A, intra and extra-cellular iron deposits (ID) encircle a dilated vein (V) in a cerebral MS plaque, Perls' method $150 x$. Panel $B$, intra and extra-cellular iron deposits (ID) encircle a dilated vein (V) in venous ulcer bed, Perls' method $80 x$

to possible release of free iron from deposits. ${ }^{4-9,13,14}$ Wenk et $a .^{7}$ and Yeoh-Ellerton ${ }^{13}$ found increased iron levels in exudates from chronic leg ulcers as compared to acute wounds. They also observed significant concentrations of metabolites from oxidative stress. 7,13

The final step of the pathogenetic chain leading to matrix disruption and ulcer development involves overexpression of matrix metallo-proteases (MMPs) that are not substantially balanced by their physiological tissue inhibitors (TIMPs). MMPs cause a substrate-specific degradation of matrix components, including collagen, elastin and laminin. Unrestricted MMP activity can lead to matrix break down and ulcer onset. ${ }^{4,15}$ Some experiments demonstrate that local iron overload may induce MMP hyper-activation through the so-called MMP iron-driven pathway. 4,16 However, the iron hypothesis does not readily explain

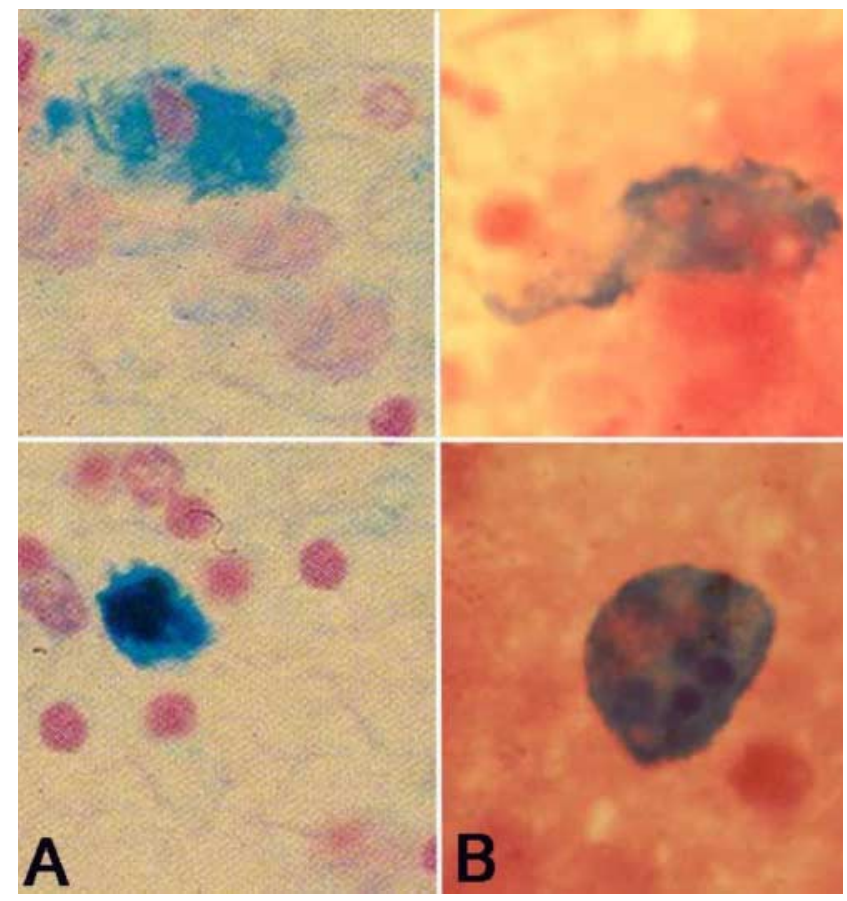

Figure 3 Panel A, top and bottom: iron-laden macrophages migrated in the cerebral extra-cellular matrix in course of MS, Perls' staining, $200 \mathrm{x}$. Panel B, top and bottom: iron-laden macrophages migrated in the extra-cellular matrix in course of CVD, Perls' staining, $400 x$ why leg iron deposits in CVD produce lesions only in some individuals. We hypothesized that such individual differences could be genetically determined, and investigated the role of the $\mathrm{C} 282 \mathrm{Y}$ and H63D mutations of the HFE gene, associated with hemochromatosis in Northern European populations. C282Y mutation significantly increases the risk of ulcer in primary CVD by more than six-fold, ${ }^{6}$ while patients carrying the $\mathrm{H} 63 \mathrm{D}$ variant have an earlier age of ulcer onset by almost 10 years. ${ }^{5}$ HFE mutations are associated with increased iron efflux from the macrophage. Our findings support the hypothesis that lesions are promoted by enhanced iron release and ROS generation. ${ }^{4-9,13,14,16}$

\section{PARALLELS BETWEEN INFLAMMATION IN CVD AND IN MS}

Clinical observations sometimes suggest alternative explanations of previous findings. During a duplex scanning examination on the carotid arteries of a 55-year-old patient with multiple sclerosis (MS), I observed an unexpected reflux from the chest into the internal jugular vein after the patient coughed involuntarily. I then noted this unusual phenomenon in other MS patients (Figure 4).

There were previous reports of a close relationship between dilated cerebral veins and inflammatory lesions In MS. Fog ${ }^{17}$ showed that the plaques of cerebral MS arise from definite segments of large epiventricular veins and that the lesions digitating out into the cerebral hemispheres also consistently evolve in a corresponding vein relationship. Putnam ${ }^{18}$ showed plaques lined with gliotic tissue containing large veins, surrounded by hematogenous pigment (Figure 2A). On a cerebral hemisphere medial aspect, a

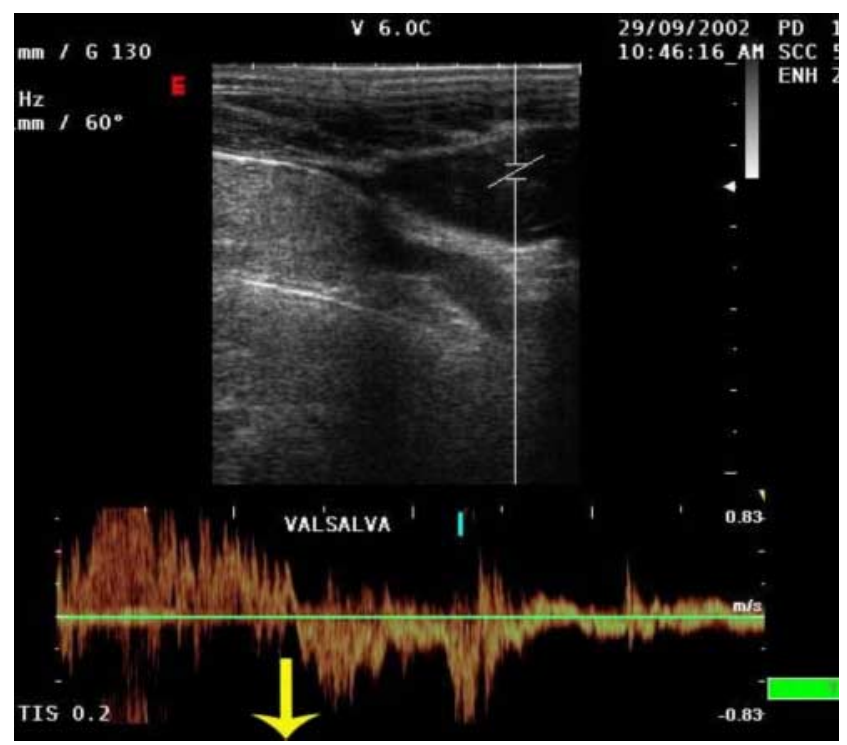

Figure 4 Reflux documented in an internal jugular vein under Valsalva in a patient affected by MS. Inversion of the flow wave from positive to negative (i.e. from chest to the head) is well apparent. 
number of vein-centred plaques spread beneath the lateral ventricular wall and surge up off of the corpus callosum under-surface. The stem and first branches of a large ventricular vein have grooved wide beds whose breadth is nearly three times that of the involved vessel diameters, a detail reminiscent of Charcot's first documentation of cerebral MS. ${ }^{19}$

Fibrin cuffs are not an exclusive finding of CVD, but are commonly visible around cerebral veins in the course of MS and today they are interpreted as ongoing reparative processes $^{20,21}$ (Figure 1B). MRI venography confirms in vivo the close relationship between the main cerebral veins and the inflammatory plaques. In 94/95 MS lesions, a central vein was visible. ${ }^{22}$ When cortical lesions occur, they arise within the territory of the principal cortical veins..$^{23,24}$ In another study, contrast MRI allowed documentation of the break-down of the blood-brain barrier (BBB). Such an injury preceded other MRI abnormalities and the clinical evidence of a new lesion. This supports the view that a defect in the BBB, and therefore inflammation, is an early and possibly crucial event in the pathogenesis of a new lesion in MS. ${ }^{25}$

Inflammation in MS is characterized by expression of adhesion molecules, ${ }^{26}$ followed by a migration of macrophages and $\mathrm{T}$-cells across the BBB. Infiltration of the matrix by macrophages, as in CVD, is considered a crucial $\operatorname{step}^{27}$ (Figure $3 \mathrm{~A}$ and $\mathrm{B}$ ). In both situations, macrophages appear with considerable intracellular iron stores due to phagocytosis of senescent erythrocyte. Iron overload in MS plaques has been demonstrated in vivo by MRI ${ }^{28}$ In addition, we observed haemosiderin in the urine of patients with active inflammation of MS (personal unpublished data).

Iron-laden macrophages carrying the HFE mutation display increased iron export, increasing the risk of generation of free iron and free radicals, possibly extending tissue lesions. ${ }^{5,6}$ A study from Australia ${ }^{29}$ suggests that C282Y-HFE mutation is increased in MS cases of North Western European origin and supports further investigations into the role of iron metabolism in the severity of MS.

As in a venous ulcer, a key determinant of tissue injury is played by MMP9. Exactly as in CVD, the over-expression of MMP9 is insufficiently counterbalanced by its tissue inhibitor TIMP-1. MMP9 can trigger leukocyte transendothelial traffic through an altered BBB, and serum active MMP9/TIMP-1 is now considered an appropriate indicator of ongoing MS inflammation. ${ }^{30}$ Despite histological findings showing haemosiderin deposits encircling the central vein of MS lesions (Figure 2A), the iron-MMP pathway of activation is not considered in MS literature.

\section{DISCUSSION}

Table 1 summarizes the iron-dependent inflammatory chain in CVD and shows impressive pathophysiological similarities with MS. The critical point of the proposed parallelism between CVD and MS involves venous haemodynamics. In CVD, altered venous haemodynamics are considered the trigger mechanism of subsequent inflammation. In contrast, altered venous haemodynamics in MS are poorly studied. The possible role of venous reflux/obstruction in cerebral and spinal veins requires

Table 1 Common findings of the inflammatory chain in CVD and MS

\begin{tabular}{lllll}
\hline Finding & CVD & References & Multiple sclerosis & References \\
\hline Altered venous haemodynamics & + & $1,4-12$ & $?$ & $17-19$ \\
Perivenous inflammation & + & $2,4,5,9,10$ & + & $17-25$ \\
erythrocyte extra-vasation & + & $4-9$ & + & $18-21,24$ \\
Haemosiderin deposits & + & $4-9$ & + & 20,21 \\
Adhesion molecules and white cells activation & + & $10-12$ & + & 26,27 \\
Macrophage migration-infiltration & + & $4-6,12$ & + & $26,27,30$ \\
T cell migration-infiltration & + & 12 & + & $26,27,30$ \\
Iron laden-macrophage & + & $4-6$ & + & 20,21 \\
MMPs hyper-activation & + & $4-7,15$ & + & 30 \\
TIMPs hypo-expression & + & $4-7,15$ & + & 30 \\
Local iron overload & + & $4-9$ & + & $18,20,21,28$ \\
Urine haemosiderin test & + & 9 & & Personal unpublished \\
HFE mutation & + & 5,6 & + & data \\
Fibrin cuff (on going reparative process) & + & 2 & + & 29 \\
& & & & 20,21
\end{tabular}


additional investigation. Earlier literature indicated that the inflammatory lesions spread counter-current to the normal venous flow direction, and the process of cerebral multiple sclerosis advances in a direction diametrically opposed to that of normal venous flow. ${ }^{17-19}$ Such circumstances should be further investigated with the help of advanced neuroimaging technology.

Although investigations on the role of iron in MS are still few, some evidence supports a pivotal role for iron in MS inflammation. The effect of manipulation of iron level was investigated in EAE, a form of induced autoimmune encephalomyelitis in mice used as an experimental model of MS. ${ }^{31}$ The incidence of EAE was $60-70 \%$ in mice with a normal iron level and in iron-overloaded mice, but $0 \%$ in iron-deficient mice. The findings suggest that iron deficiency provides protection from the development of $E^{2} E^{31}$ and also challenge traditional views on what constitutes a normal level of stored iron. ${ }^{14}$ The authors ${ }^{31}$ noted that, 'The failure of iron-deficient mice to develop EAE is impressive. Many of the pharmaceutical approaches to inhibiting EAE are less effective than iron deficiency.'

Another group ${ }^{32}$ investigated the serum concentration of soluble transferrin receptor (sTFR) in a group of MS patients. The levels were found to be significantly higher in patients with active MS, either in progressive or relapsingremitting clinical form, than in controls. Serum ferritin levels were also significantly elevated in patients affected by the active and progressive form. ${ }^{32}$ Both findings support the hypothesis above described, which proposes local iron overload as the initial signal of the inflammatory chain in MS.

Although the primum movens of MS is still elusive, these studies suggest that iron-dependent mechanisms of inflammation seen in CVD could be relevant to MS. Future work on MMPs and on iron/macrophage interactions appears especially promising. However, because of its relevant epidemiology and its easily visualized lesions, CVD is an ideal model for investigating iron mediated mechanisms of tissue injury of venous and inflammatory origin, as well as the use of deliberate induction of iron deficiency as a treatment modality.

Acknowledgments This research was supported by the Italian Ministry for the University and the Scientific Research and by the Foundation Cassa di Risparmio di Ferrara.

Competing interests None declared.

\section{REFERENCES}

1 Eklof B, Rutherford RB, Bergan JJ, et al. for the American Venous Forum International Ad Hoc Committee for Revision of the CEAP Classification. Revision of the CEAP classification for chronic venous disorders: consensus statement. J Vasc Surg 2004;40:1248-52

2 Browse NL, Burnand KG. The cause of venous ulceration. Lancet $1982 ; 2: 243-5$
3 Joshi A, Sloan P. Role of 'fibrin' cuffs in chronic nonspecific oral ulceration. Wound Repair and Regeneration 2004;12:18

4 Zamboni P, Scapoli G, Lanzara V, et al. Serum iron and MMP-9 variations in limbs affected by chronic venous disease and venous leg ulcers. Dermatol Surg 2005;31:644-9

5 Zamboni P, Izzo M, Tognazzo S, et al. The overlapping of local iron overload and HFE mutation in venous leg ulcer pathogenesis. Free Radic Biol Med 2006;40:1869-73

6 Zamboni P, Tognazzo S, Izzo M, et al. Hemochromatosis C282Y gene mutation increases the risk of venous leg ulceration. J Vasc Surg 2005;42:309-14

7 Wenk J, Foitzik A, Achterberg V, et al. Selective pick-up of increased iron by deferoxamine-coupled cellulose abrogates the iron-driven induction of matrix degrading metalloproteinase 1 and lipid peroxidation in human dermal fibroblasts in vitro: a new dressing. Concept J Invest Dermatol 2001;116:833-9

8 Ackerman Z, Seidenbaum M, Loewenthal E, Rubinow A. Overload of iron in the skin of patients with varicose ulcers. Possible contributing role of iron accumulation in progression of the disease. Arch Dermatol $1988 ; 124: 1376-8$

9 Zamboni P, Izzo M, Fogato L, Carandina S, Lanzara V. Urine haemosiderin: a novel marker to assess the severity of chronic venous disease. J Vasc Surg 2003;37:132-6

10 Coleridge Smith PD, Thomas P, Scurr JH, Dormandy JA. Causes of venous ulceration: a new hypothesis. BMJ 1988;296:1726-7

11 Takase S, Pascarella L, Lerond L, Bergan JJ, Schmid-Schonbein GW. Venous hypertension, inflammation and valve remodeling. Eur J Vasc Endovasc Surg 2004;28:484-93

12 Wilkinson LS, Bunker C, Edwards JC, Scurr JH, Smith PD. Leukocytes: their role in the etiopathogenesis of skin damage in venous disease. J Vasc Surg 1993;17:669-75

13 Yeoh-Ellerton S, Stacey MC. Iron and 8-isoprostane levels in acute and chronic wounds. J Invest Dermatol 2003;121:918-25

14 Sullivan JL. Is stored iron safe? J Lab Clin Med 2004;144:280-4

15 Herouy $\mathrm{Y}$, Mellios $\mathrm{P}$, Banderir E, et al. Inflammation in stasis dermatitis upregulates MMP-1, MMP-2 and MMP-13 expression. J Dermat Science 2001;25:198-205

16 Gurjar MV, Deleon J, Sharma RV, Bhalla RC. Role of reactive oxygen species in IL-1-stimulated sustained ERK activation and MMP-9 induction. Am J Phys 2001;281:H2568-H2574

17 Fog T. The topography of plaques in multiple sclerosis with special reference to cerebral plaques. Acta Neurol Scand 1965;15(Suppl):1-161

18 Putnam TJ. Lesions of 'encephalomyelitis'and multiple sclerosis. Venous thrombosis as the primary alteration. JAMA 1937;108:1477

19 Allen IV. The pathology of multiple sclerosis fact, fiction and hypothesis. Neuropathol Appl Neurobiol 1981;7:169

20 Adams CW. Vascular aspects of multiple sclerosis. In: A Colour Atlas of Multiple Sclerosis and Other Myelin Disorders. London. Wolfe Medical Publication, 1989:184-187.

21 Adams CW, Poston RN, Buk SJ. Pathology, histochemistry and immunocytochemistry of lesions in acute multiple sclerosis. J Neurol Sci 1989;92:291-306

22 Tan IL, van Schijndel RA, Pouwels PJ, et al. MR venography of multiple sclerosis. AJNR Am J Neuroradiol 2000;21:1039-42

23 Horowitz AL, Kaplan RD, Grewe G, White RT, Salberg LM. The ovoid lesion: a new MR observation in patients with multiple sclerosis. AJNR Am J Neuroradiol 1989;10:303-5

24 Kidd D, Barkhof F, McConnell R, Algra PR, Allen IV, Revesz T. Cortical lesions in multiple sclerosis. Brain 1999;122:17-26

25 Kermode AG, Thompson AJ, Tofts P, et al. Breakdown of the bloodbrain barrier precedes symptoms and other MRI signs of new lesions in multiple sclerosis. Pathogenetic and clinical implications. Brain 1990;113:1477-89 
26 Kuenz B, Lutterotti A, Khalil M, et al. Plasma levels of soluble adhesion molecules sPECAM-1, sP-selectin and sE-selectin are associated with relapsing-remitting disease course of multiple sclerosis. J Neuroimmunol 2005;167:143-9

27 Martino G, Adorini L, Rieckmann P, et al. Inflammation in multiple sclerosis: the good, the bad, and the complex. Lancet Neurol 2002;1:499-509

28 Haacke EM, Cheng NY, House MJ, et al. Imaging iron stores in the brain using magnetic resonance imaging. Magn Reson Imaging 2005;23:1-25

29 Rubio JP, Bahlo M, Tubridy N, et al. Extended haplotype analysis in the HLA complex reveals an increased frequency of the HFE-C282Y mutation in individuals with multiple sclerosis. Hum Genet 2004;114:573-80

30 Fainardi E, Castellazzi M, Bellini T, et al. Cerebrospinal fluid and serum levels and intrathecal production of active matrix metalloproteinase-9 (MMP-9) as markers of disease activity in patients with multiple sclerosis. Mult Scler 2006;12:294-301

31 Grant SM, Wiesinger JA, Beard JL, Cantorna MT. Iron-deficient mice fail to develop autoimmune encephalomyelitis. J Nutr 2003;133:2635-8

32 Sfagos C, Makis AC, Chaidos A, et al. Serum ferritin, transferrin and soluble transferrin receptor levels in multiple sclerosis patients. Mult Scler $2005 ; 11: 272-5$ 